\title{
Recurrent cervicocephalic artery dissection in a patient with Churg-Strauss syndrome treated with long-term corticosteroid therapy
}

\author{
Hyung Jun Kim, Soo Ryun Park, Oh Young Bang
}

Department of Neurology, Samsung Medical Center, Sungkyunkwan University School of Medicine, Seoul, Korea

Received: September 9, 2019

Revised: November 15, 2019

Accepted: November 20, 2019

Corresponding author:

Hyung Jun Kim

Department of Neurology,

Samsung Medical Center,

Sungkyunkwan University

School of Medicine, 81 Irwon-

ro, Gangnam-gu, Seoul 06351 , Korea

Tel: +82-2-3410-8238

E-mail:hhhj7.kim@samsung.com
This is an Open Access article distributed under the terms of the Creative Commons Attribution Non-Commercial License (http:// creativecommons.org/licenses/ by-nc/4.0/).

\begin{abstract}
Churg-Strauss syndrome (CSS) is known to mainly involve small vessels; thus, in the event of large-vessel disease, other causes must be taken into consideration. We report a case of CSS with recurrent arterial dissection of the cervicocephalic arteries. A 74-yearold man with CSS visited the emergency department because of sudden left hemiparesis. He had been taking corticosteroids as CSS treatment for 12 years. He had a confirmed right vertebral dissection 10 years before and a right distal internal carotid artery dissection 1 year before. As the arterial dissection occurred in the same vessel region a year before, a stent was deployed in the region. A large cervicocephalic artery dissection in patients with CSS that is well regulated with immunotherapy is likely caused by the long-term corticosteroid therapy weakening the vessel wall; therefore, replacement with the appropriate immunotherapy should be considered.
\end{abstract}

Keywords: Cerebral infarction; Churg-Strauss syndrome; Corticosteroids; Dissection

\section{INTRODUCTION}

Churg-Strauss syndrome (CSS) is a disseminated small-vessel systemic necrotizing vasculitis with extravascular granulomas that occurs exclusively among patients with asthma and tissue eosinophilia [1]. Corticosteroid is one of the most important treatments for CSS. It is used as the first-line therapy and often administered for long periods [1]. In patients with CSS, small vessels are typically involved, but medium vessels such as the coronary and middle cerebral arteries have also been reported to be involved [2,3]. However, no report has described cases of CSS involving large vessels such as the carotid artery. In cases of CSS with a large-vessel involvement, the mechanisms of stroke is unsettled because of difficulty of vessel biopsy. The cause of stroke in patients with CSS could be either the direct involvement of CSS of the large cervicocephalic vessels or secondary to a comorbidity or the adverse effects of the CSS treatment.

Herein, we report a case of CSS with recurrent arterial dissection of the large cervicocephalic arteries and discuss the possible mechanisms of stroke in patients with CSS during long-term steroid therapy. 


\section{CASE REPORT}

A 74-year-old man was admitted with sudden left hemiparesis and left-side neglect. He had a history of asthma, hypereosinophilia, mononeuritis multiplex, and paranasal sinus abnormality and was diagnosed as having CSS in accordance with the diagnostic criteria of the American College of Rheumatology [4]. Since 12 years before, when he was first diagnosed as having CSS, he had been receiving treatment with $15-\mathrm{mg} /$ day prednisolone and $100-\mathrm{mg} /$ day azathioprine. Prednisolone was continuously administered at $<20 \mathrm{mg} /$ day, except in the initial stage of the disease. Recently, he showed no worsening of symptoms related to CSS, and his peripheral eosinophil count was within the normal range of $120 /$ L. He had no risk factor of stroke, except hyperlipidemia.

The patient had multiple episodes of ischemic cerebrovas- cular events. He had a history of multiple cerebellar infarctions due to the right vertebral artery dissection 10 years before (Fig. 1A-C). A year before, he had recurrent border-zone infarcts (both internal and cortical) in the right hemisphere. Magnetic resonance angiography (MRA) revealed an occlusive lesion on the right distal internal carotid artery (ICA), which was completely recanalized on the MRA performed 6 months later, suggesting spontaneous dissection as the cause of the stroke (Fig. 1D, E). The patient denied any trauma, infection, or symptoms related to CSS during the events.

Neurological examination revealed a dense left hemiparesis, right-gaze preponderance, left-sided neglect, and dysarthria. His initial National Institutes of Health Stroke Scale (NIHSS) score was 13. The results of the routine laboratory tests, including complete blood count with high-sensitivity C-reactive protein level, chemistry, coagulation test, urine analysis,
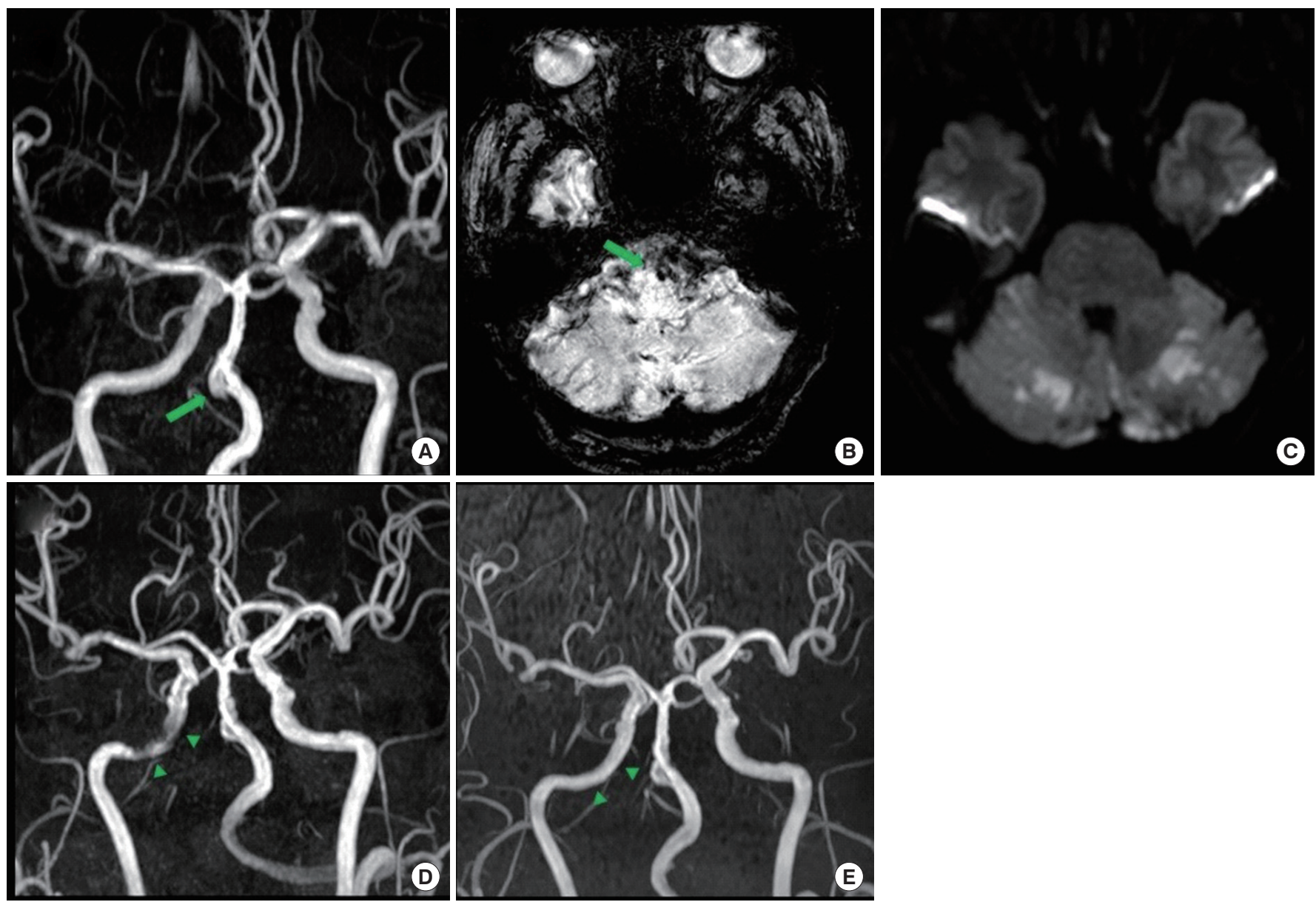

C

Fig. 1. Previous brain magnetic resonance images and magnetic resonance angiogram of brain arteries. Occlusion of the right distal vertebral artery (A: arrow) and dark intensity in the right distal vertebral artery, which represents an intramural hematoma (B: arrow). (C) Diffusion-weighted magnetic resonance image of multiple cerebellar acute infarcts affecting both cerebellar hemispheres. (D, E) Right distal internal carotid artery dissection 1 year before (D: arrowheads), showing full recovery on the magnetic resonance angiogram obtained 6 months before (E: arrowheads). 
and chest radiography, were all within the normal limits. Cardiac investigations, including 72-hour telemonitoring and transthoracic echocardiography, revealed negative results.

Brain magnetic resonance imaging revealed an infarction in the territory of the right middle cerebral artery, and MRA revealed an occlusion of the right distal ICA, which increased the suspicion of dissection recurrence (Fig. 2A-C). Emergent diagnostic angiography revealed a tapered occlusion of the right ICA with the "flame" sign, without carotid bulb involvement and highly suggestive of dissection (Fig. 2D) [5]. Owing to clinical deterioration and marked perfusion delay in the right cerebral hemisphere, a permanent stent placement was planned. By using a Prowler 14 microcatheter (Cordis Corp., Miami Lakes, FL, USA), we entered the ophthalmic segment of the distal ICA, after checking the true lumen on microcatheter angiography (Fig. 2E). We deployed the carotid wall stent 9/50 (Boston Scientific Corp., Marlborough, MA, USA) from the distal common carotid artery to the cervical ICA and subsequently performed another angiography, which confirmed no distal ICA flow (Fig. 2F). Thus, we de-
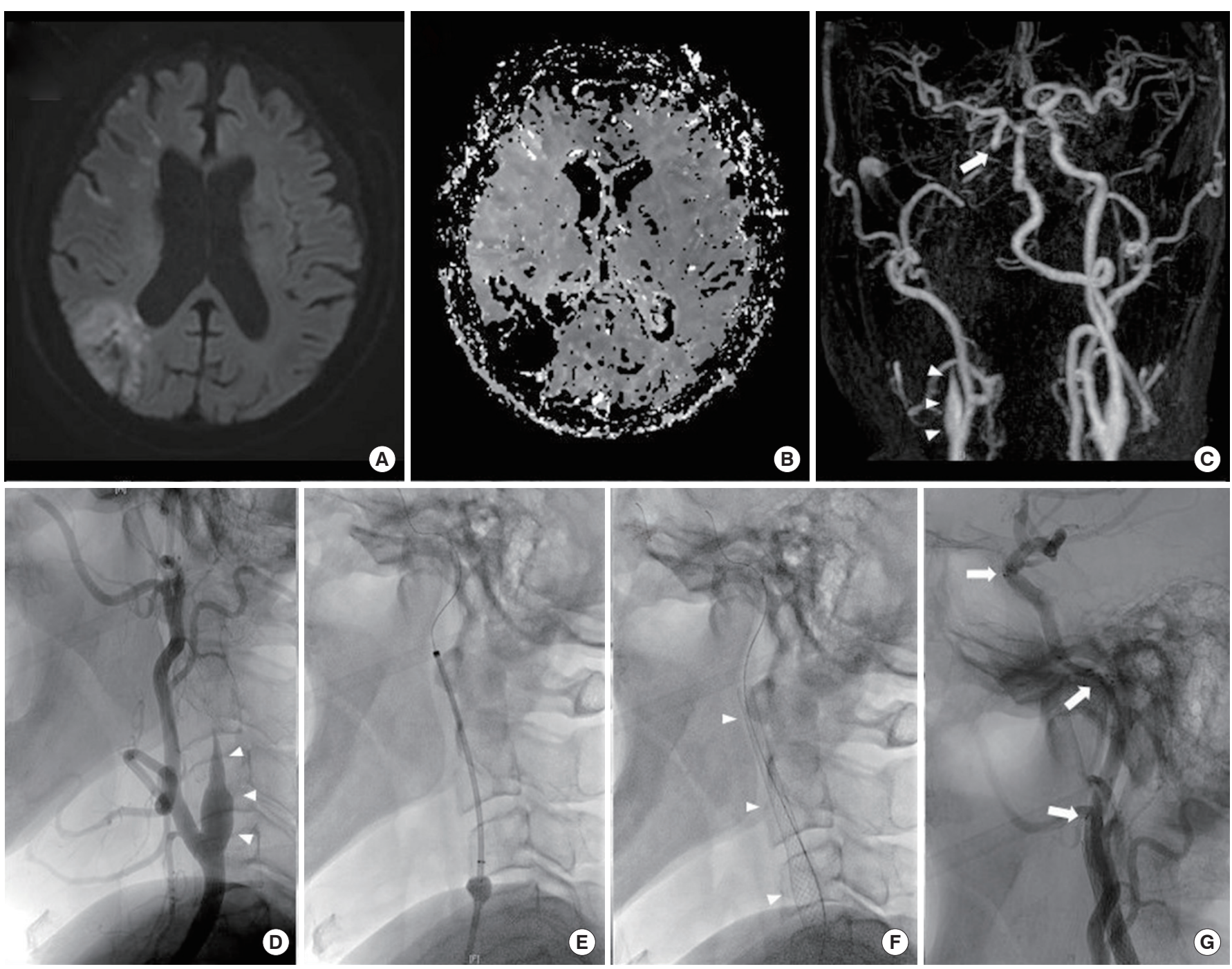

Fig. 2. (A) Brain magnetic resonance images of multiple infarctions in the territory of the right middle cerebral artery, and (B) increased time to peak in the corresponding region (perfusion map). (C) Magnetic resonance angiogram showing a tapered occlusion of the right proximal internal carotid artery (ICA) (arrowheads) and abrupt cut-off of the distal ICA (arrow), suggestive of distal ICA occlusion. suggestive of distal ICA occlusion. (D) Catheter angiogram showing a tapered occlusion of the right proximal ICA from the mid cervical region to the petrous segment (arrowheads). (E) By using a Prowler 14 microcatheter (Cordis Corp.), we entered the ophthalmic segment of the distal ICA. (F) Deployment of the carotid wall stent $9 / 50$ (Boston Scientific Corp.) from the distal common carotid artery to the cervical ICA (arrowheads). (G) Deployment of two Enterprise 4/39 stents (Codman neurovascular), one in the petrous segment and the other from the distal petrous segment to the ophthalmic segment (arrows). 
ployed the Enterprise 4/39 stent (Codman Neurovascular, Raynham, MA, USA) from the petrous ICA to the ophthalmic ICA (Fig. 2G). The final angiography images showed resolution of the right ICA occlusion with improved perfusion of the right hemisphere. The patient's NIHSS score improved to 10 points, and he was transferred to the rehabilitation department. The azathioprine therapy was maintained, and he well tolerated the reduced corticosteroid dose.

Written informed consent was obtained from the patient for the publication of this case report.

\section{DISCUSSION}

Ischemic stroke is uncommon in patients with CSS [6]. The cause of ischemic stroke is diverse. In most cases, stroke is of cardioembolic origin due to cardiac involvement of CSS [7] or small deep infarcts due to eosinophilic inflammation and necrotizing vasculitis of the capillaries or arterioles [6]. In patients with CSS, a large-vessel stroke can occur, with artery dissection as a possible cause. However, only coronary artery dissection has been reported, and cerebral artery dissection has not been reported [3]. To our knowledge, this is the first case report of recurrent spontaneous dissection of the large vessels that involved both the intracranial and extracranial cervicocephalic arteries in a patient with CSS well controlled with long-term steroid therapy.

The association of spontaneous dissection with CSS or long-term steroid therapy is largely unknown. The advent of corticosteroid treatment undoubtedly improves the overall prognosis of patients with CSS but may have deleterious effects on the cardiovascular system $[1,8,9]$. Long-term use of steroids is associated with arterial atherosclerosis and has adverse effects on arterial connective tissues $[9,10]$. In addition, it causes hypertension and hyperlipidemia, which have negative effects on blood vessels [11]. On the contrary, longterm steroid therapy can increase blood vessel fragility due to its negative effect on connective tissue strain [8]. In this regard, aortic aneurysm occurs after long-term corticosteroid therapy [8], which causes catheter-induced bilateral coronary artery dissection [12]. Arecent case report showed that multiple cervical vessel dissections occurred after 2 weeks of short-term corticosteroid therapy, suggesting indirectly that the steroid therapy could also affect the cervical artery [10].

Vessel fragility is highly likely related to the long-term corticosteroid therapy in our patient, who experienced frequent (three recurrences) spontaneous cervicocephalic dissections during long-term corticosteroid therapy, with well-controlled
CSS. In this case, we decided to switch from the corticosteroid therapy to immunotherapy. Our present case suggests that if spontaneous dissection occurs repeatedly in a patient with an autoimmune disease such as CSS, vessel fragility related to corticosteroid therapy should be suspected and the corticosteroid dose should be decreased or replaced with other immunotherapies. On the contrary, increased corticosteroid or immunotherapy dose could be considered when stroke occurs during deterioration of autoimmune disease.

\section{CONFLICTS OF INTEREST}

No potential conflict of interest relevant to this article was reported.

\section{ACKNOWLEDGMENTS}

This study was supported by the National Research Foundation of Korea (NRF) grant (No. 2018R1A2B2003489).

\section{ORCID}

Hyung Jun Kim https://orcid.org/0000-0002-7518-9739

Soo Ryun Park https://orcid.org/0000-0001-7488-6543

Oh Young Bang https://orcid.org/0000-0002-7962-8751

\section{REFERENCES}

1. Greco A, Rizzo MI, De Virgilio A, Gallo A, Fusconi M, Ruoppolo $\mathrm{G}$, et al. Churg-Strauss syndrome. Autoimmun Rev 2015;14:341-8.

2. Kang DW, Kim DE, Yoon BW, Seo JW, Roh JK. Delayed diagnosis: recurrent cerebral infarction associated with Churg-Strauss syndrome. Cerebrovasc Dis 2001;12:280-1.

3. Hunsaker JC 3rd, O'Connor WN, Lie JT. Is spontaneous dissection of the coronary artery with eosinophilia a limited form of Churg-Strauss syndrome? Arch Pathol Lab Med 1994;118:863-4.

4. Masi AT, Hunder GG, Lie JT, Michel BA, Bloch DA, Arend WP, et al. The American College of Rheumatology 1990 criteria for the classification of Churg-Strauss syndrome (allergic granulomatosis and angiitis). Arthritis Rheum 1990;33:1094-100.

5. Ozdoba C, Sturzenegger M, Schroth G. Internal carotid artery dissection: MR imaging features and clinical-radiologic correlation. Radiology 1996;199:191-8.

6. Caplan LR, Biller J. Uncommon causes of stroke. 3rd ed. 
Cambridge (UK): Cambridge University Press; 2018. p. 331-3.

7. Bhagirath KM, Paulson K, Ahmadie R, Bhalla RS, Robinson $D$, Jassal DS. Clinical utility of cardiac magnetic resonance imaging in Churg-Strauss syndrome: case report and review of the literature. Rheumatol Int 2009;29:445-9.

8. Ohara N, Miyata T, Sato O, Oshiro H, Shigematsu H. Aortic aneurysm in patients with autoimmune diseases treated with corticosteroids. Int Angiol 2000;19:270-5.

9. Bulkley BH, Roberts WC. The heart in systemic lupus erythematosus and the changes induced in it by corticosteroid therapy. A study of 36 necropsy patients. Am J Med
1975;58:243-64.

10. Chung SE, Yoon TH, Lee KM, Kim HG, Kim BJ. A case report of multiple cervical artery dissection after peripheral type facial palsy and use of steroids. BMC Neurol 2018; 18:74.

11. Keir ML, Dehghani P. Corticosteroids and spontaneous coronary artery dissection: a new predisposing factor? Can J Cardiol 2016;32:395.e7-8.

12. Miyachi H, Tanaka K, Mizuno K. Catheter-induced bilateral coronary ostium dissection in a patient with long-term steroid therapy. J Invasive Cardiol 2012;24:E305-7. 